\title{
Editorial: Advances in the Structural Elucidation and Utilization of Lignins
}

\author{
Fengxia Yue ${ }^{1 *}$ and Li Shuai ${ }^{2}$ \\ ${ }^{1}$ State Key Laboratory of Pulp and Paper Engineering, School of Light Industry and Engineering, South China University of \\ Technology, Guangzhou, China, ${ }^{2}$ College of Material Engineering, Fujian Agriculture and Forestry University, Fuzhou, China
}

Keywords: lignin, structural characterization, valorization, aromatic resource, fractionation

Editorial on the Research Topic

Advances in the Structural Elucidation and Utilization of Lignins

As one of the most abundant plant-derived aromatic resources on the planet, lignin has drawn increasing attention in the past few decades. Although remarkable progress has been made in lignin structural elucidation and utilization in recent years, lignin valorization remains the most changing topic due to the complexity and inherent heterogeneity of lignin. As an irregular and non-repeated polymer, lignin has long been recognized as the major recalcitrant that hinders the utilization of cellulose.

Normally, native lignin is a highly complex natural polymer formed primarily from three typical monolignol precursors, including $p$-coumaryl alcohol, coniferyl alcohol, and sinapyl alcohol, that differ in the methoxyl degree of the aromatic ring via a combinatorial radical coupling process. The monoligniols are then displayed as $p$-hydroxyphenyl $(\mathrm{H})$, guaiacyl $(\mathrm{G})$, and syringyl $(\mathrm{S})$ units, so called lignin structural units, that linked by several types of $\mathrm{C}-\mathrm{O}$ and $\mathrm{C}-\mathrm{C}$ linkages, including $\beta-\mathrm{O}-4, \beta-5, \beta-\beta, 5-5, \beta-1$, and $4-\mathrm{O}-5$ linkages formed during lignin biosynthesis (Figure 1). Among these linkages, $\beta-\mathrm{O}-4$ alkyl aryl ethers, accounting for $50-80 \%$ of the total linkages in native lignins, are the most predominant interunit linkages in lignin polymer. However, selective fractionation of biomass into different constituents (i.e., carbohydrates and lignin) will cause significant structural changes due to degradation and condensation reactions. The resultant condensed lignin increases the difficulty for its valorization. Therefore, efficient fractionation and characterization are two essential prerequisites of valorizing lignin. Understanding reaction mechanisms involved during lignin isolation and revealing the structure of lignin are crucial for development of new methods for lignin fractionation and valorization, especially for the full valorization of lignocellulosic biomass.

The topic "Advances in the structural elucidation and utilization of lignins" covers isolation, characterization and utilization of lignin. Here we heartily acknowledge all the contributors for their amazing works on this topic. Following are the highlights drawn from the contributions to this special topic. Understanding the structural changes during lignin isolation is important for optimizing the separation or pretreatment processes. Zhang et al. investigated the structural changes of lignin macromolecules of balsa wood during the growth stages by using double enzymatic lignin (DEL) that was isolated from balsa grown for different lengths of time. They found that the balsa lignin was a typical hardwood lignin overwhelmingly composed of C-O bonds (i.e., $\beta-\mathrm{O}-4$ linkages, 66.33-68.81/100Ar) and elevated with increasing tree-age, which is beneficial for the production of aromatic chemicals from lignin depolymerization. Li et al. reported the structural features and antioxidant performance of lignin fractionated from industrial furfural residue by an alkaline cooking process. They found that such lignin has appropriate radical scavenging capability and could be a promising antioxidant. Wang et al. examined the structural transformation during deep eutectic solvent (DES) treatments of enzymatic mild acidolysis lignin (EMAL) and a series of $\beta-\mathrm{O}-4$ lignin model compounds. They demonstrated that $\beta-\mathrm{O}-4$ linkages, existing in either real lignin or model compounds, could be cleaved by DES (ChCl/LA). The cleavage of $\beta-\mathrm{O}-4$ linkages of EMAL led to the decrease of molecular weights and the rise of hydroxyl groups, which would be beneficial for developing an efficient fractionation process. Hong et al. investigated the structural changes of alkali lignin under choline chloride/ formic acid (ChCl/FA) DES pretreatment, and revealed the lignin structural transformation during the DES 


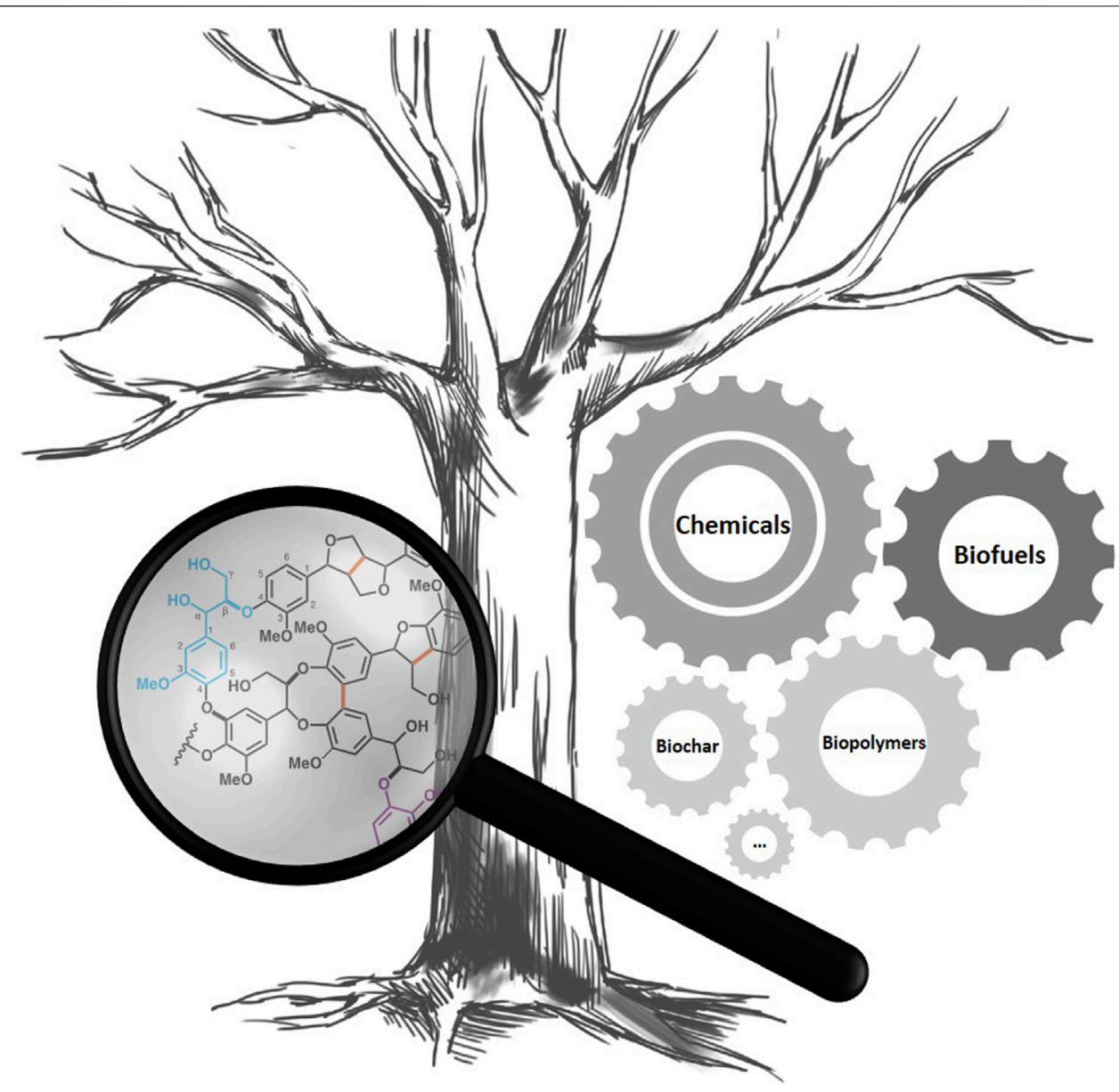

FIGURE 1 | Lignin structure and valorization.

pretreatment process, which provided new insights into preparation of homogeneous lignin with low molecular weights. Lignin model compounds always play an essential role in lignin characterization and mechanism studies. Yue et al. developed an efficient synthesis method for pinoresinol, an important lignin dimeric model compound and a high-value monolignol-derived lignan, by using 5-bromoconiferyl alcohol to suppress the undesired side-reactions by substitution at the C-5 position. Yang et al. examined the hydrognolysis of phenethyl phenyl ether (PPE) catalyzed by $\mathrm{Pd}$, $\mathrm{Ru}$, and Pt supported on carbon or $\gamma-\mathrm{Al}_{2} \mathrm{O}_{3}$ and proposed a novel strategy to monitor the ongoing reactions with in situ ${ }^{1} \mathrm{H}$ NMR and GC-MS. Wang and Ben evaluated the aging mechanism of bio-oils by model compound studies. They found that small acids, aldehydes, and HMF were the most active molecules and acidity was an important factor affecting the aging process of the bio-oils. Incorporating lignin into polymers is one of the most promising way of utilizing lignin, and the properties of the lignin-based polymers highly depend on the structural features of lignin. Wang et al. examined the effects of cosolvent-enhanced lignocellulosic fractionation (CELF) pretreatment severity on lignin structure of CELF-extracted lignin, and analyzed the influence of the structure on the mechanical properties of CELF lignin-based polyurethane. Finally, Dong et al. described the performance of porous carbons produced from lignin nanoparticles (LNPs) by two different protocols, and found the combination of hydrothermal carbonization and pyrolysis would improve the specific surface areas and total pore volumes of LNPs derived graphene-like porous carbons.

\section{AUTHOR CONTRIBUTIONS}

All authors listed have made a substantial, direct, and intellectual contribution to the work and approved it for publication.

Conflict of Interest: The authors declare that the research was conducted in the absence of any commercial or financial relationships that could be construed as a potential conflict of interest.

Copyright (C) 2021 Yue and Shuai. This is an open-access article distributed under the terms of the Creative Commons Attribution License (CC BY). The use, distribution or reproduction in other forums is permitted, provided the original author(s) and the copyright owner(s) are credited and that the original publication in this journal is cited, in accordance with accepted academic practice. No use, distribution or reproduction is permitted which does not comply with these terms. 